NASA/TM-1998-208744

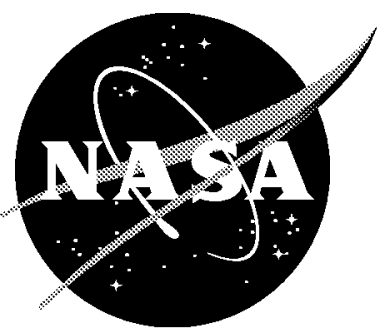

\title{
Predictive Feedback and Feedforward Control for Systems with Unknown Disturbances
}

Jer-Nan Juang and Kenneth W. Eure

Langley Research Center, Hampton, Virginia 
The NASA STI Program Office... in Profile

Since its founding, NASA has been dedicated to the advancement of aeronautics and space science. The NASA Scientific and Technical Information (STI) Program Office plays a key part in helping NASA maintain this important role.

The NASA STI Program Office is operated by Langley Research Center, the lead center for NASA's scientific and technical information. The NASA STI Program Office provides access to the NASA STI Database, the largest collection of aeronautical and space science STI in the world. The Program Office is also NASA's institutional mechanism for disseminating the results of its research and development activities. These results are published by NASA in the NASA STI Report Series, which includes the following report types:

- TECHNICAL PUBLICATION. Reports of completed research or a major significant phase of research that present the results of NASA programs and include extensive data or theoretical analysis. Includes compilations of significant scientific and technical data and information deemed to be of continuing reference value. NASA counterpart and peer-reviewed formal professional papers, but having less stringent limitations on manuscript length and extent of graphic presentations.

- TECHNICAL MEMORANDUM. Scientific and technical findings that are preliminary or of specialized interest, e.g., quick release reports, working papers, and bibliographies that contain minimal annotation. Does not contain extensive analysis.

- CONTRACTOR REPORT. Scientific and technical findings by NASA-sponsored contractors and grantees.
- CONFERENCE PUBLICATION. Collected papers from scientific and technical conferences, symposia, seminars, or other meetings sponsored or co-sponsored by NASA.

- SPECIAL PUBLICATION. Scientific, technical, or historical information from NASA programs, projects, and missions, often concerned with subjects having substantial public interest.

- TECHNICAL TRANSLATION. Englishlanguage translations of foreign scientific and technical material pertinent to NASA's mission.

Specialized services that complement the STI Program Office's diverse offerings include creating custom thesauri, building customized databases, organizing and publishing research results. .. even providing videos.

For more information about the NASA STI Program Office, see the following:

- Access the NASA STI Program Home Page at http://www.sti.nasa.gov

- E-mail your question via the Internet to help@sti.nasa.gov

- Fax your question to the NASA STI Help Desk at (301) 621-0134

- Phone the NASA STI Help Desk at (301) 621-0390

- Write to: NASA STI Help Desk NASA Center for AeroSpace Information 7121 Standard Drive Hanover, MD 21076-1320 


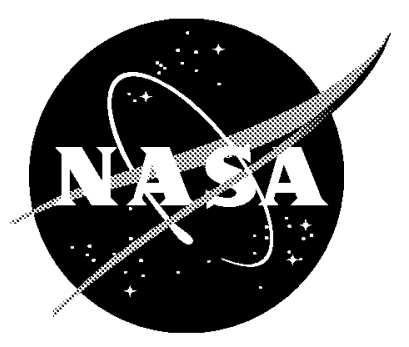

\section{Predictive Feedback and Feedforward Control for Systems with Unknown Disturbances}

Jer-Nan Juang and Kenneth W. Eure

Langley Research Center, Hampton, Virginia

National Aeronautics and

Space Administration

Langley Research Center

Hampton, Virginia 23681-2199 
Available from:

NASA Center for AeroSpace Information (CASI) 7121 Standard Drive

Hanover, MD 21076-1320

(301) 621-0390
National Technical Information Service (NTIS) 5285 Port Royal Road

Springfield, VA 22161-2171

(703) 605-6000 


\title{
Predictive Feedback and Feedforward Control for Systems with Unknown Disturbances
}

\author{
Jer-Nan Juang *and Kenneth W. Eure ${ }^{\dagger}$ \\ NASA Langley Research Center \\ Hampton, VA 23681
}

\section{Abstract}

Predictive feedback control has been successfully used in the regulation of plate vibrations when no reference signal is available for feedforward control. However, if a reference signal is available it may be used to enhance regulation by incorporating a feedforward path in the feedback controller. Such a controller is known as a hybrid controller. This paper presents the theory and implementation of the hybrid controller for general linear systems, in particular for structural vibration induced by acoustic noise. The generalized predictive control is extended to include a feedforward path in the multi-input multi-output case and implemented on a single-input single-output test plant to achieve plate vibration regulation. There are cases in acoustic-induced vibration where the disturbance signal is not available to be used by the hybrid controller, but a disturbance model is available. In this case the disturbance model may be used in the feedback controller to enhance performance. In practice, however, neither the disturbance signal nor the disturbance model is available. This paper presents the theory of identifying and incorporating the noise model into the feedback

*Principal Scientist, Structural Dynamics Branch.

$\dagger$ Aerospace Technologist, Structural Acoustics Branch, and also Graduate Student, Department of Electrical Engineering, VPI\&SU 
controller. Implementations are performed on a test plant and regulation improvements over the case where no noise model is used are demonstrated.

\section{Introduction}

Recent advancements in microprocessor technology have made it possible to successfully apply predictive feedback control theory ${ }^{1-11}$ to regulate acoustically induced vibrations without a reference signal or disturbance model. ${ }^{12}$ However, if information about the disturbance is known, this information may be used in the controller design to improve the ability of the controller to regulate the plant. ${ }^{13}$ The internal noise model has been shown to enhance the performance of the Linear Quadratic Regulator $(\mathrm{LQR})^{14}$ if the disturbance states can be estimated. Predictive control may likewise be extended to take advantage of disturbance information. The disturbance information may be in the form of a noise model or the input disturbance signal may be available. Given the present and past values of the disturbance, a predictor may be used to estimate future disturbance values when there is coherence in the disturbance signal.

Given the present and past values of the disturbance signal, a finite-difference equation may be determined to model the correlation in the disturbance signal. With this finitedifference model, future disturbance values may be estimated and incorporated into the hybrid controller to enhance performance. In the case of single frequency disturbances, plant regulation may be dramatically improved. As the disturbance bandwidth increases, the regulation enhancement obtained by the disturbance predictions diminishes.

If the disturbance signal is not measurable or available for feedforward, one important question arises if the disturbance signal can ever be recovered or estimated from the control input and output signals. An innovative approach has been developed ${ }^{15}$ using the concept 
of a deadbeat observer to identify a system with unknown periodic disturbances. Another approach has also been introduced ${ }^{16}$ using the concept of multi-step output prediction for identification of the system and periodic disturbances.

The objective of this paper is to present hybrid predictive control methods including feedback and feedforward that can be used for systems with unknown disturbances. This paper begins with addressing the problems of system identification and disturbance estimation. With unknown periodic disturbances, a new approach is developed to characterize the relationship between the control signal and the output measurement signal. The approach begins with the finite-difference equation in the $z$-domain. It is shown that the transfer function from the control input to the measurement output can be fully recovered without need of knowing the disturbances. The disturbance-induced output is shown to be embedded in the observer Markov parameters for the finite-difference model describing the correlation between the control signal and the output signal. Furthermore, the disturbance-induced output can be computed and predicted. Several hybrid predictive control techniques are presented including feedback plus feedforward and feedback with embedded feedforward. These techniques are verified and compared using a simple test for reducing structural vibration induced by acoustic noise.

\section{System Identification and Disturbance Estimation}

A generic block diagram of the closed-loop control system is shown in Fig. 1. There are two fundamental steps involved in the closed-loop system. The first step is to identify a mathematical model. The second step is to use the identified model to design a controller. In this paper, the finited difference model is used for the process of system identfication as well as predictive control designs. The controller may contain the disturbance information in 
the form of a feedforward transfer function or the disturbance information may be embedded in the feedback control parameters. If feedforward is used, the controller needs to have access to the disturbance signal. If the disturbance information is embedded in the feedback control parameters, it will be proven that the controller needs no measurement of the disturbance signal.

The input/output relationship of a linear system is commonly described by a finite difference model. ${ }^{18}$ Given a system with $r_{c}$ control inputs, $r_{d}$ disturbance inputs, and $m$ outputs, the finite difference model (FDM) for the $r_{c} \times 1$ input $u(k), r_{d} \times 1$ input $d(k)$ and the $m \times 1$ output $y(k)$ at time $k$ is

$$
\begin{aligned}
y(k)= & \alpha_{1} y(k-1)+\alpha_{2} y(k-2)+\cdots+\alpha_{p} y(k-p) \\
& +\beta_{0} u(k)+\beta_{1} u(k-1)+\beta_{2} u(k-2)+\cdots+\beta_{p} u(k-p) \\
& +\gamma_{0} d(k)+\gamma_{1} d(k-1)+\gamma_{2} d(k-2)+\cdots+\gamma_{p} d(k-p)
\end{aligned}
$$

This simply means that the current output can be predicted by the past input and output time histories. The finite difference model is also often referred to as the ARX model where AR refers to the AutoRegressive part and X refers to the eXogeneous part. The coefficient matrices, $\alpha_{i}$ of $m \times m, \beta_{i}$ of $m \times r_{c}$, and $\gamma_{i}$ of $m \times r_{d}$ for $i=0,1, \ldots, p$ are commonly referred to as the observer Markov parameters $(\mathrm{OMP})^{19-22}$ or ARX parameters, because they are closely related to the deadbeat observer.

The equivalent version of Eq. (1) in the $z$-domain is

$$
\alpha(z) y(z)=\beta(z) u(z)+\gamma(z) d(z)
$$

where $y(z), u(z)$, and $d(z)$ are the $z$-transform of the data sequences $y(k), u(k)$, and $d(k)$, 
respectively, and

$$
\begin{aligned}
& \alpha(z)=I_{m}-\alpha_{1} z^{-1}-\alpha_{2} z^{-2}-\cdots-\alpha_{p} z^{-p} \\
& \beta(z)=\beta_{0}+\beta_{1} z^{-1}+\beta_{2} z^{-2}+\cdots+\beta_{p} z^{-p} \\
& \gamma(z)=\gamma_{0}+\gamma_{1} z^{-1}+\gamma_{2} z^{-2}+\cdots+\gamma_{p} z^{-p}
\end{aligned}
$$

where $I_{m}$ is an $m \times m$ identity matirx.

\subsection{Single Disturbance Input}

For simplicity, let us assume that there is only one disturbance, i.e., $r_{d}=1$, applied to the system at a certain location. Furthermore, the disturbance signal $d(k)$ for $k=1,2, \ldots, \ell$ is known to be periodic. For a periodic signal (i.e., correlated signal), there exists a finitedifference model such that

$$
d(k)=\eta_{1} d(k-1)+\eta_{2} d(k-2)+\cdots+\eta_{n_{d}} d\left(k-n_{d}\right)
$$

where $n_{d}$ is the model order which is twice the number of frequencies in the disturbance signal and $\eta_{i}\left(i=1,2, \ldots, n_{d}\right)$ are the constant scalars weighting the past disturbance signal. The maximum disturbance frequency should be less than the Nyquest frequency which is one half of the sampling frequency. Equation (6) in the $z$-domain becomes

$$
\eta(z) d(z)=0
$$

where

$$
\eta(z)=1-\eta_{1} z^{-1}-\eta_{2} z^{-2}-\cdots-\eta_{n_{d}} z^{-n_{d}}
$$

Premultiplying Eq. (2) by $\eta(z)$ and noting Eq. (7) yields

$$
\eta(z) \alpha(z) y(z)=\eta(z) \beta(z) u(z)
$$


or equivalently

$$
\tilde{\alpha}(z) y(z)=\tilde{\beta}(z) u(z)
$$

where

$$
\begin{aligned}
& \tilde{\alpha}(z)=\eta(z) \alpha(z)=I_{m}-\tilde{\alpha}_{1} z^{-1}-\tilde{\alpha}_{2} z^{-2}-\cdots-\tilde{\alpha}_{p} z^{-p-n_{d}} \\
& \tilde{\beta}(z)=\eta(z) \beta(z)=\tilde{\beta}_{0}+\bar{\beta}_{1} z^{-1}+\tilde{\beta}_{2} z^{-2}+\cdots+\tilde{\beta}_{p} z^{-p-n_{d}}
\end{aligned}
$$

Note that the order of $\tilde{\alpha}$ and $\tilde{\beta}$ is $p+n_{d}$ whereas the order of $\alpha$ and $\beta$ is $p$. Equation (10) indicates that, for a periodic disturbance, there exists a finite-difference model mapping exactly from the control input to the measurement output without the need of knowing the disturbance signal. The question arises if this is true for the general case with multiple disturbance inputs.

\subsection{Multiple Disturbance Inputs}

Let us now look at the case where $r_{d}>1$, i.e., multiple disturbance inputs. First, reformulate Eq. (2) to become

$$
\alpha(z) y(z)=\beta(z) u(z)+e(z)
$$

where

$$
e(z)=\gamma(z) d(z)
$$

Here $e(z)$ is an $m \times 1$ vector, $\gamma(z)$ is an $m \times r_{d}$ matrix, and $d(z)$ is a $r_{d} \times 1$ vector. When $d(z)$ is the $z$-transform of a periodic signal (i.e., correlated signal) $d(k)$, there exists an equation identical to Eq. (7) except that the quantity $\eta(z)$ in this case is a $r_{d} \times r_{d}$ matrix. As a result, there should exist a finite-difference equation such that

$$
\mathcal{E}(z) e(z)=\mathcal{E}(z) \gamma(z) d(z)=\mathcal{E}^{\prime}(z) \eta(z) d(z)=0
$$


where $\mathcal{E}(z)$ is an $m \times m$ matrix and $\mathcal{E}^{\prime}(z)$ is an $m \times r_{d}$ matrix. Equation (15) implies that

$$
\mathcal{E}(z) \gamma(z) d(z)-\mathcal{E}^{\prime}(z) \eta(z) d(z)=0
$$

or, if $d(z) \neq 0$,

$$
\left[\begin{array}{ll}
\mathcal{E}(z) & \mathcal{E}^{\prime}(z)
\end{array}\right]\left[\begin{array}{c}
\gamma(z) \\
-\eta(z)
\end{array}\right]=0
$$

Given $\gamma(z)$ of $m \times r_{d}$ and $\eta(z)$ of $r_{d} \times r_{d}$, both $\mathcal{E}(z)$ of $m \times m$ and $\mathcal{E}^{\prime}(z)$ of $m \times r_{d}$ can be found by solving the null space of the $\left(m+r_{d}\right) \times r_{d}$ matrix

$$
\left[\begin{array}{c}
\gamma(z) \\
-\eta(z)
\end{array}\right]
$$

Equation (17) shows that there always exists a matrix $\mathcal{E}(z)$ such that Eq. (15) is satisfied. Let $\mathcal{E}(z)$ be defined as

$$
\mathcal{E}(z)=I-\mathcal{E}_{1} z^{-1}-\mathcal{E}_{2} z^{-2}-\cdots-\mathcal{E}_{p^{\prime}} z^{-p^{\prime}}
$$

Each quantity $\mathcal{E}_{i}\left(i=1,2, \ldots, p^{\prime}\right)$ is an $m \times m$ matrix. The integer $p^{\prime}$ is the order of the finite-difference model. Assume that the order of the disturbance signal is $n_{d}$ (twice the number of disturbance frequencies). This implies that the determinant of Eq. (19) can have the maximum number, $n_{d}$, of roots. As a result, the relationship between the integers $p^{\prime}$ and $n_{d}$ should be

$$
p^{\prime} m \geq n_{d}=\text { twice the number of disturbance frequencies }
$$

Premultiplying Eq. (2) by $\mathcal{E}(z)$ and noting Eq. (15) yield

$$
\mathcal{E}(z) \alpha(z) y(z)=\mathcal{E}(z) \beta(z) u(z)
$$

or equivalently

$$
\bar{\alpha}(z) y(z)=\bar{\beta}(z) u(z)
$$


where

$$
\begin{aligned}
& \bar{\alpha}(z)=\mathcal{E}(z) \alpha(z)=I_{m}-\bar{\alpha}_{1} z^{-1}-\bar{\alpha}_{2} z^{-2}-\cdots-\bar{\alpha}_{p} z^{-p-p^{\prime}} \\
& \bar{\beta}(z)=\mathcal{E}(z) \beta(z)=\bar{\beta}_{0}+\bar{\beta}_{1} z^{-1}+\bar{\beta}_{2} z^{-2}+\cdots+\bar{\beta}_{p} z^{-p-p^{\prime}}
\end{aligned}
$$

The order of $\bar{\alpha}$ and $\bar{\beta}$ is $p+p^{\prime}$ whereas the order of $\alpha$ and $\beta$ is $p$. Equation (22) indicates that, for any periodic disturbance, there exists a finite-difference model mapping exactly from the control input to the measurement output without need of knowing the disturbance signal.

\subsection{System Identification}

If the matrix $\mathcal{E}(z)$ is nonsingular, Eqs. (2) and (22) produce the same system transfer function from the control input to the measurement output, i.e.,

$$
\mathcal{G}(z)=\bar{\alpha}(z)^{-1} \bar{\beta}(z)=\alpha(z)^{-1} \beta(z)
$$

It is known that the inverse transform of the transfer function $\mathcal{G}(z)$ forms a sequence of system Markov parameters. Equation (25) thus implies that both $\bar{\alpha}(z)^{-1} \bar{\beta}(z)$ and $\alpha(z)^{-1} \beta(z)$ produce the same sequence of system Markov parameters. It is quite easy to compute the coefficient matrices $\bar{\alpha}_{1}, \ldots, \bar{\alpha}_{p+p^{\prime}}$ for $\bar{\alpha}$ and $\bar{\beta}_{0}, \bar{\beta}_{1}, \ldots, \bar{\beta}_{p+p^{\prime}}$ for $\bar{\beta}$ from input and output sequences, $u(k)$ and $y(k)$ for $k=1,2, \ldots, \ell$ (see Refs. 18-22). The coefficient matrices

$\bar{\alpha}_{1}, \ldots, \bar{\alpha}_{p+p^{\prime}}$ and $\bar{\beta}_{0}, \bar{\beta}_{1}, \ldots, \bar{\beta}_{p+p^{\prime}}$ do not represent the actual observer Markov parameters, because they include the information of the periodic disturbance applied to the system. Nevertheless, they should produce the system Markov parameters for the map from the control input to the measurement output because of Eq. (25). The system Markov parameters can be computed recursively as shown in Refs. 18-22. From the computed system Markov parameters, the actual observer Markov parameters $\alpha_{1}, \ldots, \alpha_{p}$ for $\alpha$ in Eq. (3) and $\beta_{0}, \ldots, \beta_{p}$ 
for $\beta$ in Eq. (4) can be easily computed. The same results as shown in this section have also been proven using the concept of the deadbeat observer ${ }^{15}$ or multi-step output prediction. ${ }^{16}$

\subsection{Disturbance Identification}

Equation (13) can then be used to estimate the characteristics of the disturbance inputs, i.e,

$$
e(z)=\alpha(z) y(z)-\beta(z) u(z)
$$

Using the signal $e(k)$ that represents the inverse $z$-transform of $e(z)$, the $\mathcal{E}(z)$ shown in Eq. (19) can be determined to form the following finite-difference equation

$$
e(k)=\mathcal{E}_{1} e(k-1)+\mathcal{E}_{2} e(k-2)+\cdots+\mathcal{E}_{p^{\prime}} e\left(k-p^{\prime}\right)
$$

for the disturbance-induced output error. The state-space representation of Eq. (27) is

$$
\left[\begin{array}{c}
e(k) \\
e(k-1) \\
\vdots \\
e\left(k-p^{\prime}+1\right)
\end{array}\right]=\left[\begin{array}{ccccc}
\mathcal{E}_{1} & \mathcal{E}_{2} & \cdots & \mathcal{E}_{p^{\prime}-1} & \mathcal{E}_{p^{\prime}} \\
I & 0 & \cdots & 0 & 0 \\
\vdots & \vdots & \ddots & \vdots & \vdots \\
0 & 0 & \cdots & I & 0
\end{array}\right]\left[\begin{array}{c}
e(k-1) \\
e(k-2) \\
\vdots \\
e\left(k-p^{\prime}+1\right) \\
e\left(k-p^{\prime}\right)
\end{array}\right]
$$

The eigenvalues of the matrix

$$
\left[\begin{array}{ccccc}
\mathcal{E}_{1} & \mathcal{E}_{2} & \cdots & \mathcal{E}_{p^{\prime}-1} & \mathcal{E}_{p^{\prime}} \\
I & 0 & \cdots & 0 & 0 \\
\vdots & \vdots & \ddots & \vdots & \vdots \\
0 & 0 & \cdots & I & 0
\end{array}\right]
$$

provide the frequency information of the periodic disturbance applied to the system.

For the case where the disturbance input signal is white and random, the signal may be considered as having an infinite number of frequencies, i.e., $p^{\prime} \rightarrow \infty$. For random disturbance inputs, the computed $\bar{\alpha}$ and $\bar{\beta}$ satisfying Eq. (22) are still valid, as long as the integer $p^{\prime}$ is chosen sufficiently large. The system Markov parameters computed from $\bar{\alpha}$ and $\bar{\beta}$ should approach the true values. 


\section{Generalized Predictive Control (GPC)}

It is the goal of the system identification technique to determine the observer Markov parameters (OMP) based on input and output data. The OMP may be estimated using batch least squares, recursive least squares, or any other appropriate system identification technique (Ref. [11]). If the OMP of the plant is known, the future plant outputs may be predicted using a recursive relationship as shown in Ref. [11], i.e.,

$$
y_{p+q}(k)=\mathcal{T}_{c} u_{p+q}(k)+\mathcal{B} u_{p}(k-p)+\mathcal{A} y_{p}(k-p)+\mathcal{D} d_{p}(k-p)+\mathcal{T}_{d} d_{p+q}(k)
$$

Here the vectors $y_{p+q}(k)$ and $y_{p}(k-p)$ are defined as

$$
\begin{gathered}
y_{p+q}(k)=\left[\begin{array}{c}
y(k) \\
y(k+1) \\
\vdots \\
y(k+p+q-1)
\end{array}\right] \\
y_{p}(k-p)=\left[\begin{array}{c}
y(k-p) \\
y(k-p+1) \\
\vdots \\
y(k-1)
\end{array}\right]
\end{gathered}
$$

and $u_{p+q}(k)$ and $d_{p+q}(k)$ are similar to $y_{p+q}(k)$ with $y$ replaced by $u$ and $d$, respectively. The vectors $u_{p}(k-p)$ and $d_{p}(k-p)$ are similar to $y_{p}(k-p)$ with $y$ replaced by $u$ and $d$, respectively. The matrices $\mathcal{T}_{c}$ and $\mathcal{A}$ are

$$
\begin{aligned}
\mathcal{T}_{c}= & {\left[\begin{array}{cccc}
\beta_{0} & & & \\
\beta_{0}^{(1)} & \beta_{0} & & \\
\vdots & \vdots & \ddots & \\
\beta_{0}^{(p+q-1)} & \beta_{c 0}^{(p+q-2)} & \cdots & \beta_{0}
\end{array}\right] } \\
\mathcal{A}= & {\left[\begin{array}{cccc}
\alpha_{p} & \alpha_{(p-1)} & \cdots & \alpha_{1} \\
\alpha_{p}^{(1)} & \alpha_{p-1}^{(1)} & \cdots & \alpha_{1}^{(1)} \\
\vdots & \vdots & \ddots & \vdots \\
\alpha_{p}^{(p+q-1)} & \alpha_{p-1}^{(p+q-1)} & \cdots & \alpha_{1}^{(p+q-1)}
\end{array}\right] }
\end{aligned}
$$


The matrix $\mathcal{T}_{d}$ is similar to $\mathcal{T}_{c}$ with $\beta$ replaced by $\gamma$, and $\mathcal{B}$ and $\mathcal{D}$ are similar to $\mathcal{A}$ with $\alpha$ replaced by $\beta$ and $\gamma$, respectively. In Eq. (30), $p$ is the integer related to the system order, $q$ is an intermediate time step, and the prediction horizon is $h_{p}=p+q-1$. The quantity $y_{p+q}(k)$ is the vector containing the predicted future plant outputs, whereas $u_{p+q}(k)$ is the vector containing the future control inputs yet to be determined, and $d_{p+q}(k)$ is the vector containing the future disturbance inputs yet to be predicted. Also $y_{p}(k-p)$ is the vector containing the past plant outputs, $u_{p}(k-p)$ is the vector containing the past control inputs, and $d_{p}(k-p)$ is the vector containing the past disturbance inputs.

The GPC algorithm is based on system output predictions over a finite horizon known as the prediction horizon. In order to predict the future plant outputs, some assumptions need to be made about the future control inputs and the future disturbance inputs. In determining the future control inputs, it is assumed that control is applied only over a finite horizon known as the control horizon. Beyond the control horizon, the control input is assumed to be zero. In the GPC algorithm, the control horizon is always equal to or less than the prediction horizon. In addition to the horizons, a control penalty is introduced to limit the control effort and stabilize the closed loop system. The cost function to be minimized in the GPC algorithm is.

$$
\begin{aligned}
J(k) & =y_{p+q}^{T}(k) y_{p+q}(k)+u_{p+q}^{T}(k) \lambda u_{p+q}(k) \\
& =\sum_{j=0}^{p+q-1}\left\{[y(k+j)]^{T}[y(k+j)]+[u(k+j)]^{T} \lambda[u(k+j)]\right\}
\end{aligned}
$$

In Eq.(31), $y(k+j)$ is the plant output vector, $u(k+j)$ is the control input vector, and $\lambda$ is the control penalty scalar. For simplicity, both prediction horizon and control horizon are set from 0 to $p+q-1$ and the control penalty $\lambda$ is assumed to be a positive scalar rather than a matrix. Minimizing Eq.(31) with respect to $u_{p+q}(k)$ and solving for $u_{p+q}(k)$ will give 
the control sequence to be applied to the plant. The first $r$ values of the control sequence are applied to the $r$ control inputs, the remainder is discarded, and a new control sequence is calculated the next time step. In order to carry out the above process, the future plant outputs $y(k), y(k+1), \ldots, y(k+p+q-1)$ must be predicted. In the regulation problem, the desired plant output is zero. Inserting Eq.(30) into Eq.(31), minimizing with respect to $u_{p+q}(k)$ and taking the first $r$ rows results in

$$
\begin{aligned}
u(k)= & \text { the first } r \text { rows of }\left[-\left(\mathcal{T}_{c}^{T} \mathcal{T}_{c}+\lambda I\right)^{-1} \mathcal{T}_{c}^{T}\right] \times \\
& {\left[\mathcal{B} u_{p}(k-p)+\mathcal{A} y_{p}(k-p)+\mathcal{D} d_{p}(k-p)+\mathcal{T}_{d} d_{p+q}(k)\right] }
\end{aligned}
$$

where $I$ is an identity matrix. When $\lambda=0$, the closed-loop system will be unstable for nonminimum systems because the matrix $\mathcal{T}_{c}$ is rank deficient. The quantity $\lambda$ must be carefully tuned to make the system stable.

The formulation given in Eq.(32) assumes that the control horizon is equal to the prediction horizon. Nevertheless, the control horizon may be chosen to be less than the prediction horizon resulting in a more stable and sluggish regulator. This is achieved by reducing the matrix $\mathcal{T}_{c}$ in Eq. (32) to become

$$
\mathcal{T}_{c}=\left[\begin{array}{cccc}
\beta_{0} & & & \\
\beta_{0}^{(1)} & \beta_{0} & & \\
\vdots & \vdots & \ddots & \\
\beta_{0}^{(q)} & \beta_{0}^{(q-1)} & \cdots & \beta_{0} \\
\beta_{0}^{(q+1)} & \beta_{0}^{(q)} & \cdots & \beta_{0}^{(1)} \\
\vdots & \vdots & \vdots & \vdots \\
\beta_{0}^{(p+q-1)} & \beta_{0}^{(p+q-2)} & \cdots & \beta_{0}^{\left(h_{c}\right)}
\end{array}\right]
$$

The control sequence determined by using Eq. (33) in Eq. (32) is for a shorter control horizon, i.e. $h_{c}<p+q-1$. Beyond the control horizon the control input is assumed to be zero.

The formulation given in Eq. (32) differs from that given in Ref. [2-3] in two ways. First, the current control computed by Eq. (32) will be applied at the next time step rather 
than at the present time step as required in Ref. [2-3]. This is important in implementation because the present formulation allows time to perform computations. Second, the controller coefficients are calculated using an intuitively recursive relationship, ${ }^{11}$ rather than solving the Diophantine equation for future predictions. ${ }^{2-3}$ By adjusting the control horizon, the prediction horizon, and the control cost, Eq. (32) may be tuned to yield the best results for a given regulation problem.

Equation (32) includes a feedforward path. If the disturbance signal is measurable, then it is placed in the $d_{p}(k-p)$ vector. This vector contains the last $p$ disturbance measurements. If there is correlation in the disturbance measurements such as the periodic signal, then this correlation may be used to estimate future disturbance values and fill the $d_{p+q}(k)$ vector. If there is no correlation in the disturbance signal, then the last term in Eq. (32) is dropped. Given that correlation does exist, a finite difference model may be used together with past disturbance measurements to predict future disturbance values. The finite-difference model is shown in the following

$$
d(k)+\eta_{1} d(k-1)+\eta_{2} d(k-2)+\ldots+\eta_{n_{d}} d\left(k-n_{d}\right)=0
$$

In Eq. (34), $n_{d}$ is the disturbance order and $\eta_{j}\left(j=1,2, \ldots, n_{d}\right)$ are $n_{d} \times n_{d}$ coefficient matrices. In order to include the last term in Eq. (32), we need the future disturbance signal that can be estimated by recursively solving Eq. (34) for future values of the disturbance signal based on past disturbance measurements. As a result, the $j$ th prediction of the disturbance signal may be calculated by

$$
d(k+j)=\eta_{1}^{(j)} d(k-1)+\eta_{2}^{(j)} d(k-2)+\ldots+\eta_{n_{d}}^{(j)} d\left(k-n_{d}\right)
$$

The index $j$ will go from 0 to the prediction horizon $p+q-1$. The future disturbance signals will then be estimated and the last term of Eq. (32) may be used to enhance performance. 
In practice, the disturbance signal is generally not available. The conventional design strategy is to increase the closed-loop system damping via control feedback using the input/output transfer function. The unknown disturbances are treated as input uncertainties. In most of cases, they are considered as white, Gaussian, and random noises. The closed-loop feedback thus designed may be stable but may not satisfy the performance requirements. However, it has been proven in Eq. (22) that the unknown periodic disturbances are embedded in the observer Markov parameters (OMP) which can be identified. With the OMP identified, a new approach is introduced to design a feedback law that includes an embedded forward to enhance the performance. The multi-output prediction formulation for Eq. (22) is identical to Eq. (30) with last two terms dropped. All the terms related to the unknown disturbances are embedded in the coefficient matrices $\mathcal{A}$ and $\mathcal{B}$ with the expense of increasing the number of OMP to include the order of the disturbances. The matrix $\mathcal{T}_{c}$ remains unchanged because it represents the control input/output map. Therefore, if it is not possible to measure the disturbance signal, the control law with embedded forward, Eq. (32), becomes

$$
\begin{array}{r}
u(k)=\text { the first } r \text { rows of }\left[-\left(\mathcal{T}_{c}^{T} \mathcal{T}_{c}+\lambda I\right)^{-1} \mathcal{T}_{c}^{T}\right] \times \\
{\left[\mathcal{B} u_{p+p^{\prime}}\left(k-p-p^{\prime}\right)+\mathcal{A} y_{p+p^{\prime}}\left(k-p-p^{\prime}\right)\right]}
\end{array}
$$

where $p^{\prime}$ is the order of the unknown periodic disturbances.

\section{$5 \quad$ Experimental Results}

The GPC algorithm with feedforward and a noise predictor was implemented on a Texas Instrument C-30 chip. The plant to be regulated is shown in Fig. 2. The box shown in Fig. 2 is made of plexiglass with an aluminum plate on top. The disturbance enters the plant 
through the loud speaker located at the bottom of the plexiglass box. The control input is applied to the plant through the piezo mounted on the bottom center of the aluminum plate. The plant output to be regulated is the accelerometer signal taken at the top center of the aluminum plate.

A block diagram of the plant and control system is shown in Fig. 3. In Fig. 3 the disturbance $d$ is band limited to $1 \mathrm{KHz}$ and enters the plant at point 2. The plant output is the accelerometer signal taken at point 3 . The control input is applied to the plant at point 1 of Fig. 3. Low pass filters and amplifiers were used where appropriate. The controller has a sample rate of $2.5 \mathrm{KHz}$. It includes the filtered and amplified accelerometer signal $y$, and the disturbance measurement $d$, and the control signal $u$.

Since the implementation in this study is not adaptive, we must first estimate the observer Markov parameters (OMP or ARX) of the plant in order to design a controller. This is accomplished by applying two independent white noise signals (band limited to $1 \mathrm{Kz}$ ), to point 2 of Fig. 2 and at $u$. With both these random inputs applied, the system output $y$ is measured. The two input data vectors and the one output vector are then used to approximate a finite-difference model (FDM) of the system. It is important to note that the FDM represents both the plexiglass box and the filters and amplifiers used in the controller loop. The form of the model is that of Eq. (1) which in turns yields Eq. (30). The coefficient matrices in Eq. (30) were then used to find the GPC controller coefficients shown in Eq. (32).

Figure 4 shows the autospectrum of the accelerometer signal $y$ before control (gray line), and after control (black line). The feedback law is based on a 12th order identified model with the weighting $\lambda=0.001$. The black solid line in Fig. 4 is the spectrum of the accelerometer signal when both feedforward and feedback are applied, this is known as 
hybrid control. The dotted line in Fig. 4 was obtained with feedback only. The disturbance applied to the plant was band limited white noise to $1000 \mathrm{~Hz}$ and thus no finite-difference model can be used to predict future disturbances. Therefore the last term in Eq. (32) was dropped.

Figure 5 shows the advantage gained by including the last term of Eq. (32) when possible. In this case, both band limited white noise to $1000 \mathrm{~Hz}$ and a sine wave disturbance of $800 \mathrm{~Hz}$ are applied to the plant through the speaker. In Fig. 5, the noise model was formed based on a 2nd order FDM obtained by performing a system identification on the disturbance signal. The resulting FDM and the past disturbance measurements were then used to predict the future disturbance values and incorporate the last term of Eq. (32). As seen in Fig. 5, this greatly attenuated the accelerometer signal at the disturbance frequency. However, the performance of the controller suffers at the upper end of the spectrum due to the large amount of control energy applied at $800 \mathrm{~Hz}$.

For the case of unknown periodic disturbances, a large increase in regulation may be obtained by performing the system identification in the presence of the disturbance. By doing this, a disturbance model is implicitly incorporated into the identified observer Markov parameters.

Figure 6 illustrates the disturbance model obtained by performing the system identification with the disturbance on. The figure is a pole/zero plot of the transfer function which describes the dynamics between the control input and accelerometer output. Both plots represent the same system (same system Markov parameters), however, the bottom plot contains the disturbance model. In this case, the disturbance was a $200 \mathrm{~Hz}$ sine wave. The plant has a mode at $300 \mathrm{~Hz}$ and was modeled as a 10 th order system. Note the pole 
zero cancellation in the bottom plot. This plot has the same system Markov parameters as the top plot, but has different observer Markov parameters.

Figure 7 shows the ability of a feedback controller with an embedded noise model to cancel a sine wave disturbance. The feedback law is based on a 20 th order identified model with the weighting $\lambda=0.001$. The disturbance in Fig. 7 is a $300 \mathrm{~Hz}$ tone. Since this is a resonant frequency of the plant, regulation may be performed with little control effort. The advantage of using the embedded noise model may be seen by comparing the dotted line to the solid black line. Figure 7 illustrates the ability of feedback control to greatly attenuate a periodic disturbance without the need of a reference source as in feedforward control.

Multiple sine waves may also be modeled in the observer Markov parameters (OMP), returned by the system identification. Figure. 8 illustrates the performance of a broadband controller which was designed using the OMP containing the disturbance information of a 50 th order model with the weighting $\lambda=0.001$. The disturbance signal entering the plant of Fig. 8 was the sum of a $300 \mathrm{~Hz}$ sine wave, a $1100 \mathrm{~Hz}$ sine wave, and band limited white noise to $1000 \mathrm{~Hz}$. When the system identification was performed with the disturbance on, the resulting OPM contained the information of the sine wave disturbances. As can be seen by comparing the solid line to the dotted line in Fig. 8, the embedded noise model greatly improved regulation at the frequencies of both sine wave disturbances.

The periodic disturbances in both Fig. 7 and 8 were at resonant frequencies of the plant. If the disturbance is at an off resonant frequency, regulation may still be performed if the control actuator has sufficient authority. The disturbance in Fig. 9 was a $800 \mathrm{~Hz}$ tone plus band limited white noise to $1000 \mathrm{~Hz}$. As can be seen in Fig. 9, control of the broad band resonant response is obtained along with a reduction of the off resonance $800 \mathrm{~Hz}$ tone. 
To compare feedback plus embedded disturbance model with feedback plus feedforward, the same noise and disturbance characteristics for Fig. 5 are used. Figure 11 illustrates the regulation performance of GPC with a 14th order system. The model was determined based on input and output data taken in the presence of white noise band limited to 1000 Hz plus an $800 \mathrm{~Hz}$ tone. As can be seen from Fig. 11, the controller did not completely cancel the $800 \mathrm{~Hz}$ tone due to the low system order. This results from the fact that there are not enough OMP coefficients to model both the system and disturbance characteristics. In practice, when the disturbance propagates through the plant to the accelerometer, disturbance information will be contaminated by the measurement noise. As a result, an increase in system order is required. Figure 12 compares the performance of a 20 th order controller to that of a 30th order controller. All other parameters are the same as in Fig. 11. There is an increase in regulation with an increase in system order.

Figure 13 shows broadband results using the GPC controller. Here band limited white noise was applied as the disturbance. The black line is the autospectrum of the accelerometer signal when the hybrid controller of Eq. (4) was used without the last term. It is of interest to compare the result obtained using feedback only, (dark gray line) with that of feedback with an embedded noise model, (dotted line). In both cases no feedforward was used. However, the system identification was performed in the presence of the disturbance for the dotted line. For the dark gray line, the system identification was done with the disturbance off. By having the disturbance on while gathering input and output data, the resulting system identification will incorporate some information about how the disturbance propagates through the plant to the accelerometer. The controller design will use this information to increase regulation as can be seen in when comparing the dark gray line to the dotted line in Fig. 13. 


\section{Concluding Remarks}

It is well known that feedforward control does indeed enhance feedback control. Designing feedback control based on the transfer function alone will not perform as well as hybrid control for any linear systems. This paper has shown that feedforward control can be implemented explicitly or implicitly. Explicit implementation requires identification of a disturbance model. A new approach is presented to show that the characteristics of unknown periodic disturbances are predictable and its induced vibration is controllable. In other words, the propagation of the disturbance through the system is observable and controllable. Given a sufficiently large system order, a system model can be identified including an embedded disturbance model. The states of the identified model are observable but not controllable. When the identified model is reduced to the minimum order, the reduced model provides the exact transfer function describing the control input to system output. This means that the system Markov parameters (i.e., pulse response function) are preserved. The disturbanceinduced output can be controlled using either predictive feedback alone or feedback plus feedforward. In fact, predictive feedback alone can perform as well as the combination of feedback and feedforward as evidenced by the experimental results. The key reason is that the predictive feedback controller is capable of implicitly identifying and incorporating the disturbance model into the control loop. The disadvantage is that the identified model is larger than the true system model to accommodate unknown disturbances. Note that any random disturbance can be treated as periodic disturbances with a sufficiently large number of frequencies. As a summary, the main contribution of this paper is the development of practical control methods for linear systems with unknown periodic disturbances. These methods have been theoretically proven and experimentally verified. 


\section{$7 \quad$ References}

1. Clarke, D.W., "Self-tuning Control of Nonminimum-phase Systems," Automatica, Vol. 20, No. 5, pp. 501-517, 1984.

2. Clarke, D.W., Mohtadi, C., and Tuffs, P.S., "Generalized Predictive Control - Part I. The Basic Algorithm," Automatica, Vol. 23, No. 2, pp. 137-148, 1987.

3. Clarke, D.W., Mohtadi, C., and Tuffs, P.S., "Generalized Predictive Control - Part II. Extensions and Interpretations," Automatica, Vol. 23, No. 2, pp. 149-160, 1987.

4. Dong,Y., "Extended Horizon Predictive Control," Ph.D Thesis, Case Western Reserve University, 1992.

5. Mosca, E., Optimal, Predictive, and adaptive Control, Prentice-Hall, 1995.

6. Wang, W., Henriksen, H., "Direct Adaptive Generalized Predictive Control," Proceedings of the American Control Conference, Chicago, U.S.A., pp. 2402-2406, 1992.

7. Wang, W., "A direct adaptive generalized predictive control algorithm for MIMO systems," International Journal of Control, Vol. 60, No. 6, pp. 1371-1381, 1994.

8. Bialasiewicz, J.T., Horta, L.G., and Phan, M., "Identified Predictive Control," Proceedings of the American Control Conference, Baltimore, Maryland, June 1994.

9. Phan, M. Q., and Juang, J.-N., "Predictive Controllers for Feedback Stabilization," Journal of Guidance, Control, and Dynamics, Vol. 21, No. 5, Sept.-Oct., 1998, pp. $747-753$. 
10. Goodzeit, N. and Phan, M. Q., "System and Periodic Disturbance Identification for Feedforward-Feedback Control of Flexible Spacecraft," Proceedings of the 35th Aerospace Sciences Meeting and Exhibit, Reno, Nevada, January 1997.

11. Juang, J.-N., and Phan, M. Q., "Deadbeat Predictive Controllers," Journal of Chinese (Taiwanese) Society of Mechanical Engineers, Vol. 19, No. 1, Jan.-Feb. 1998, pp. $25-37$.

12. Eure, K. W., and Juang, J.-N., "Broadband Noise Control Using Predictive Techniques," NASA Technical Memorandum 110320, January 1997.

13. Ellite, S.J., Sutton, T.J., Rafaely, B., and Johnson, M., "Design of Feedback Controllers using a Feedforward Approach," Active 95, Newport Beach, CA, USA, 1995.

14. Dorato, P., Abdallah, C., and Cerone, V., Linear-Quadratic Control, An Introduction, Prentice-Hall, 1995.

15. Goodzeit, N. E., and Phan, M. Q., "Exact System Identification in the Presence of Completely Unknown Periodic Disturbances," Department of Mechanical and Aerospace Engineering Technical Report No. 2096, Princeton University, Jan. 1997, New Jersey 08544 .

16. Phan, M. Q., Goodzeit, N. F., and Juang, J.-N., "Identification of System and Periodic Disturbance," 1997 ASME Design Engineering Technical Conferences, DETC97/VIB4256, Proceedings of DECTC '97, September 14-17, 1997, Sacramento, California.

17. Favier, G., Dubois, D., "A review of k-Step-ahead Predictors," Automatica, Vol. 26,. No. 1, pp.75-84, 1990. 
18. Juang, J.-N., Applied System Identification, Prentice-Hall, 1994.

19. Chen, C.-W., Huang, J.-K., Phan, M. Q. and Juang, J.-N., "Integrated System Identification and Modal State Estimation for Control of Large Flexible Space Structures," Journal of Guidance, Control, and Dynamics, Vol. 15, No. 1, pp. 88-95, Jan.-Feb. 1992.

20. Phan, M. Q., Juang, J.-N., and Hyland, D. C., "On Neural Networks in Identification and Control of Dynamic Systems," Wave Motion, Intelligent Structures, and Nonlinear Mechanics edited by S. Guran and D. Inman, World Scientific, Massachusetts, USA, 1995, pp. 194-225.

21. Juang, J.-N., Phan, M. Q., Horta, L.G., and Longman, R.W., "Identification of Observer/Kalman Filter Markov Parameters: Theory and Experiments," Journal of Guidance, Control, and Dynamics, Vol. 16, No. 2, March-April 1993, pp. 320-329.

22. Phan, M. Q., Horta, L.G., Juang, J.-N., and Longman, R.W., "Improvement of Observer/Kalman Filter Identification (OKID) by Residual Whitening," Journal of Vibrations and Acoustics, Vol. 117, April 1995, pp. 232-239. 


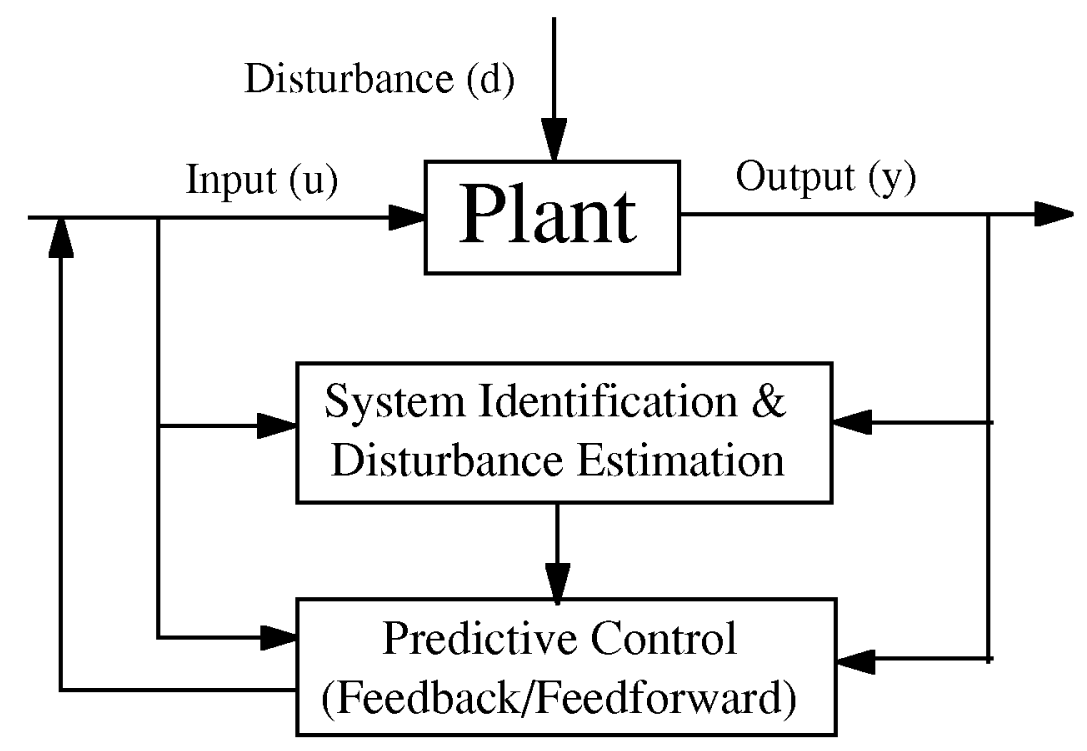

Figure 1: Generic block diagram of a closed-loop system

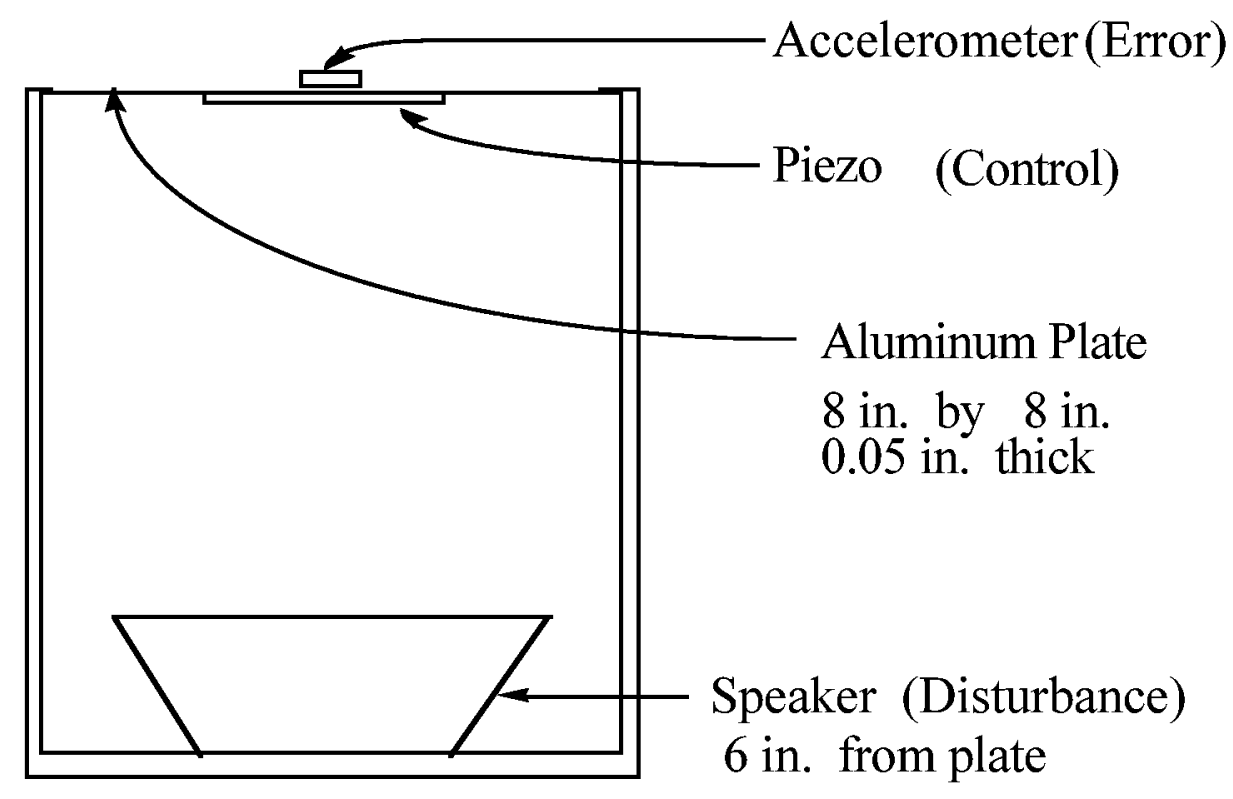

Figure 2: Plant to be Regulated 


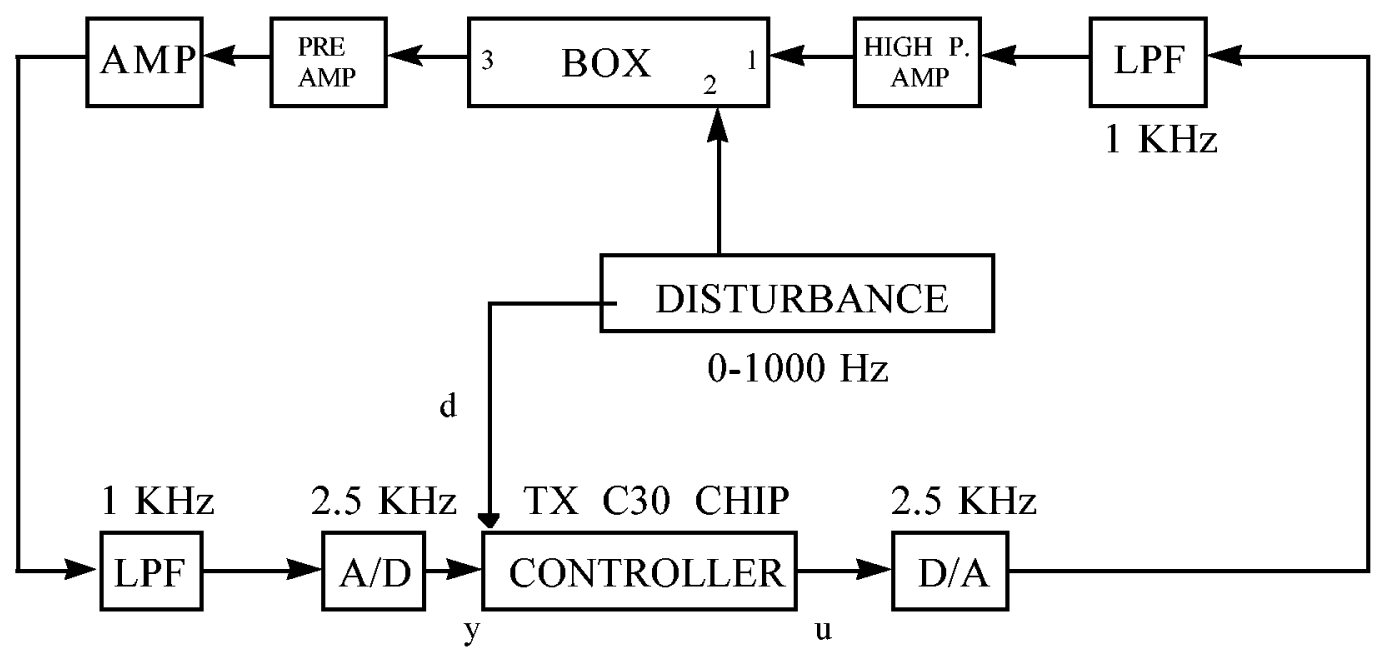

1.) Control signal goes to PZT

LPF - low pass filter acuator

2.) Disturbance enters the plant from the HIGH P. AMP - high power amplifiє speaker..

3.) Error is picked up by accelerometer

Sample rate is $2.5 \mathrm{KHz}$

Figure 3: Block Diagram of Control System 


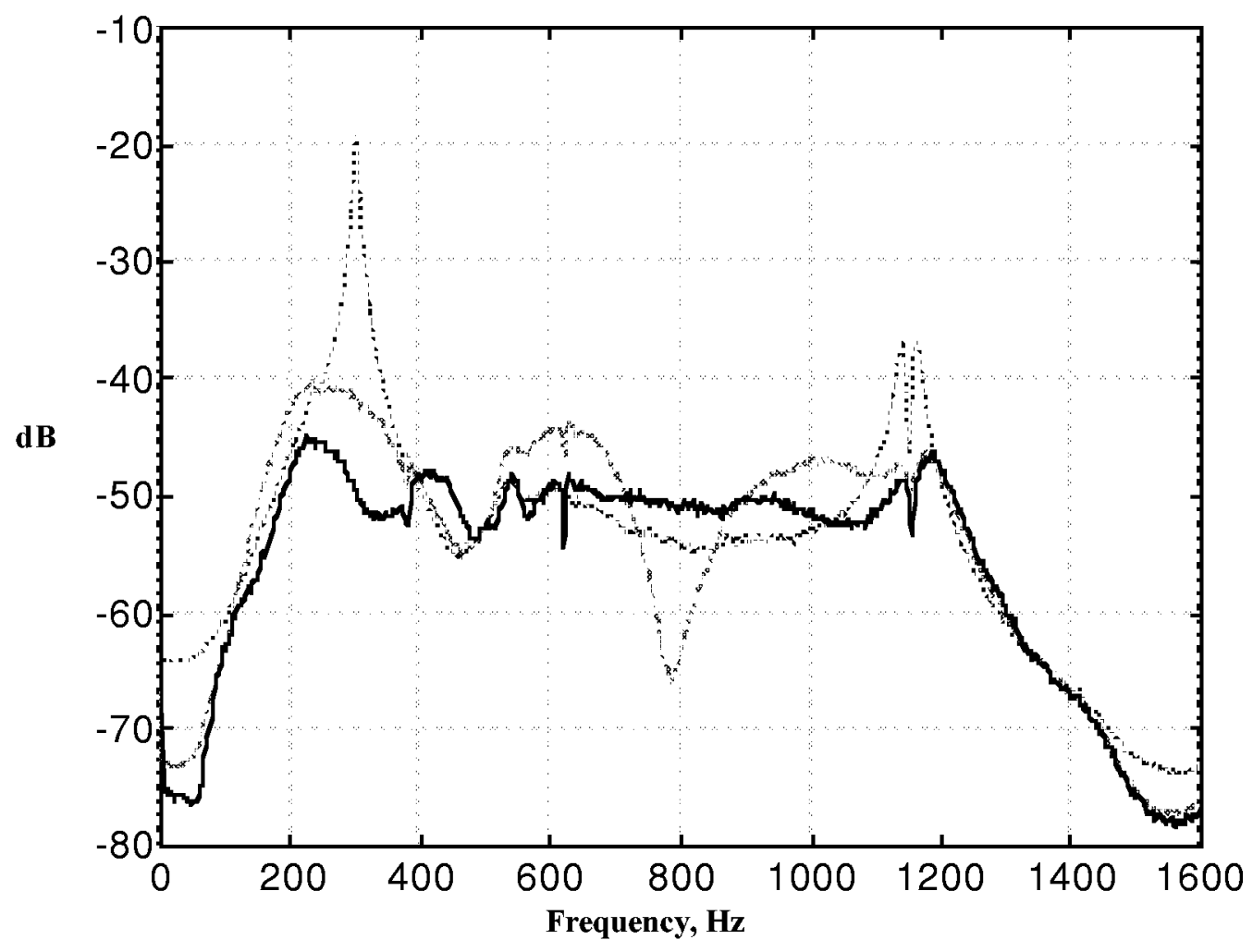

Figure 4: Autospectrum of plant output without control (dotted line), feedback only (dot dash line), and with hybrid control (solid line), 12 th order system, $2.5 \mathrm{KHz}$ sample rate. 


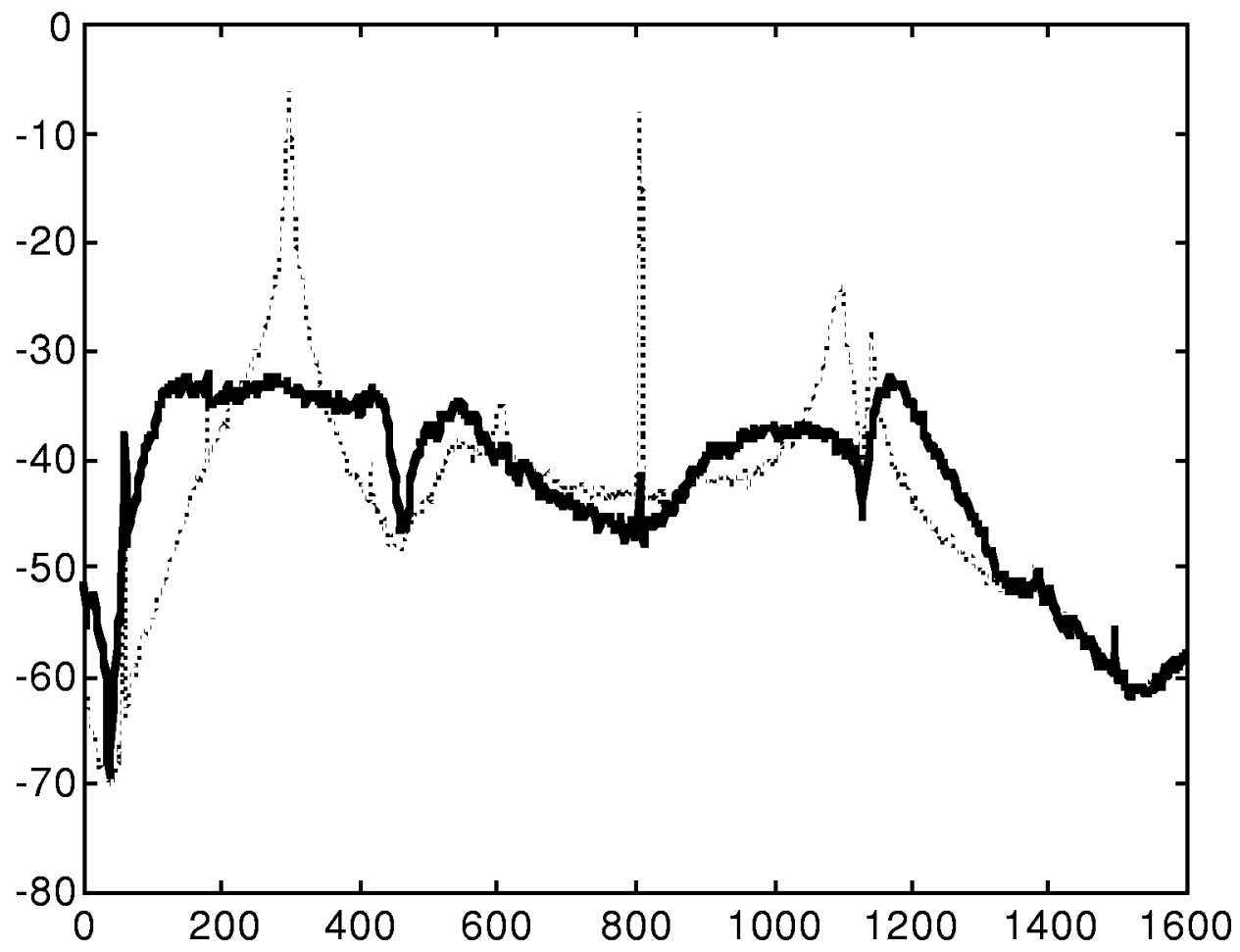

Figure 5: Autospectrum of plant output without control (dotted line) and with hybrid control plus noise predictor (solid line), 12 th order system, $2.5 \mathrm{KHz}$ sample rate. 

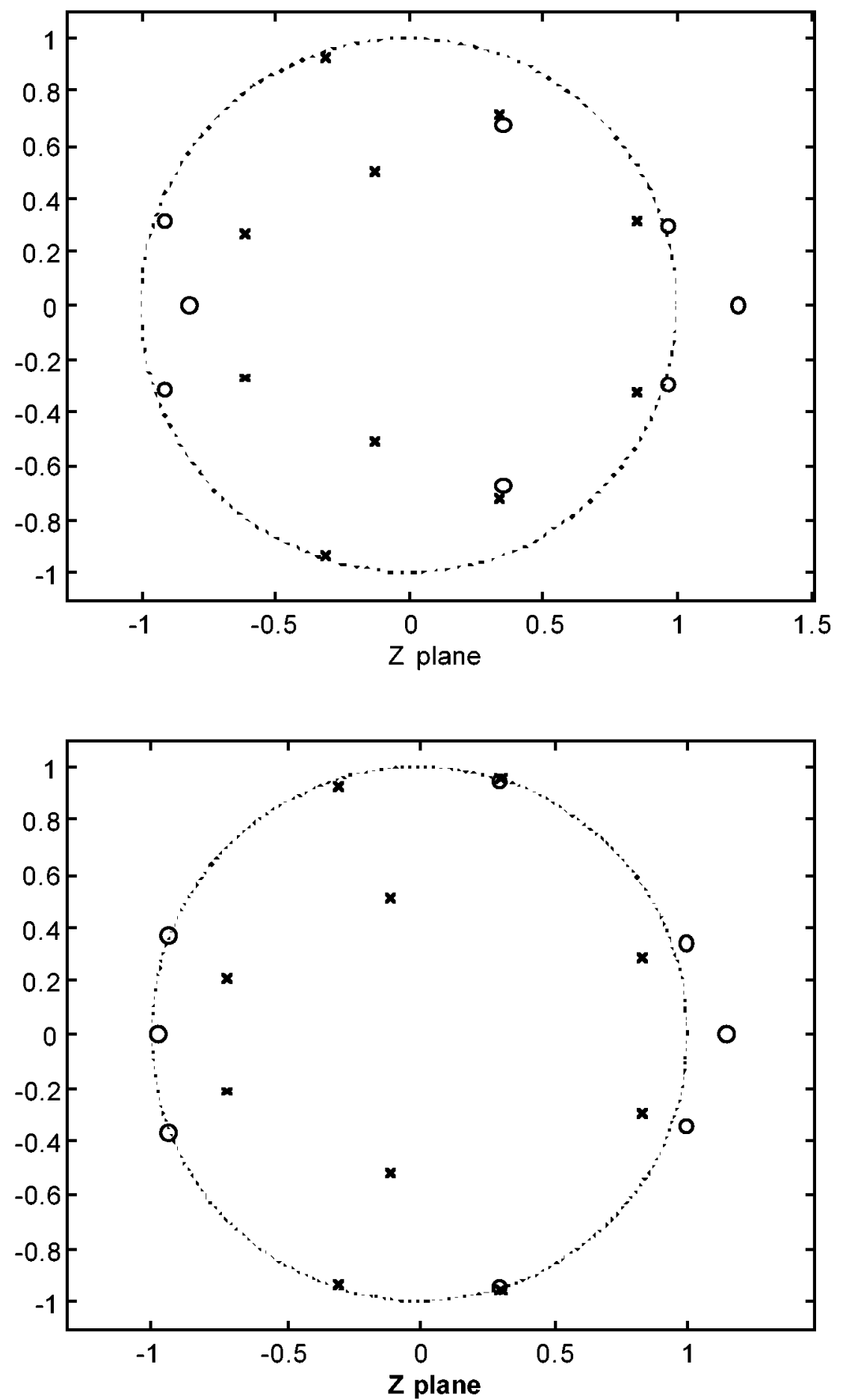

Figure 6: Pole Zero plots obtained with disturbance off (top plot) and disturbance on (bottom plot), 10th order system model, $1 \mathrm{KHz}$ sampling rate, $200 \mathrm{~Hz}$ sine wave disturbance. 


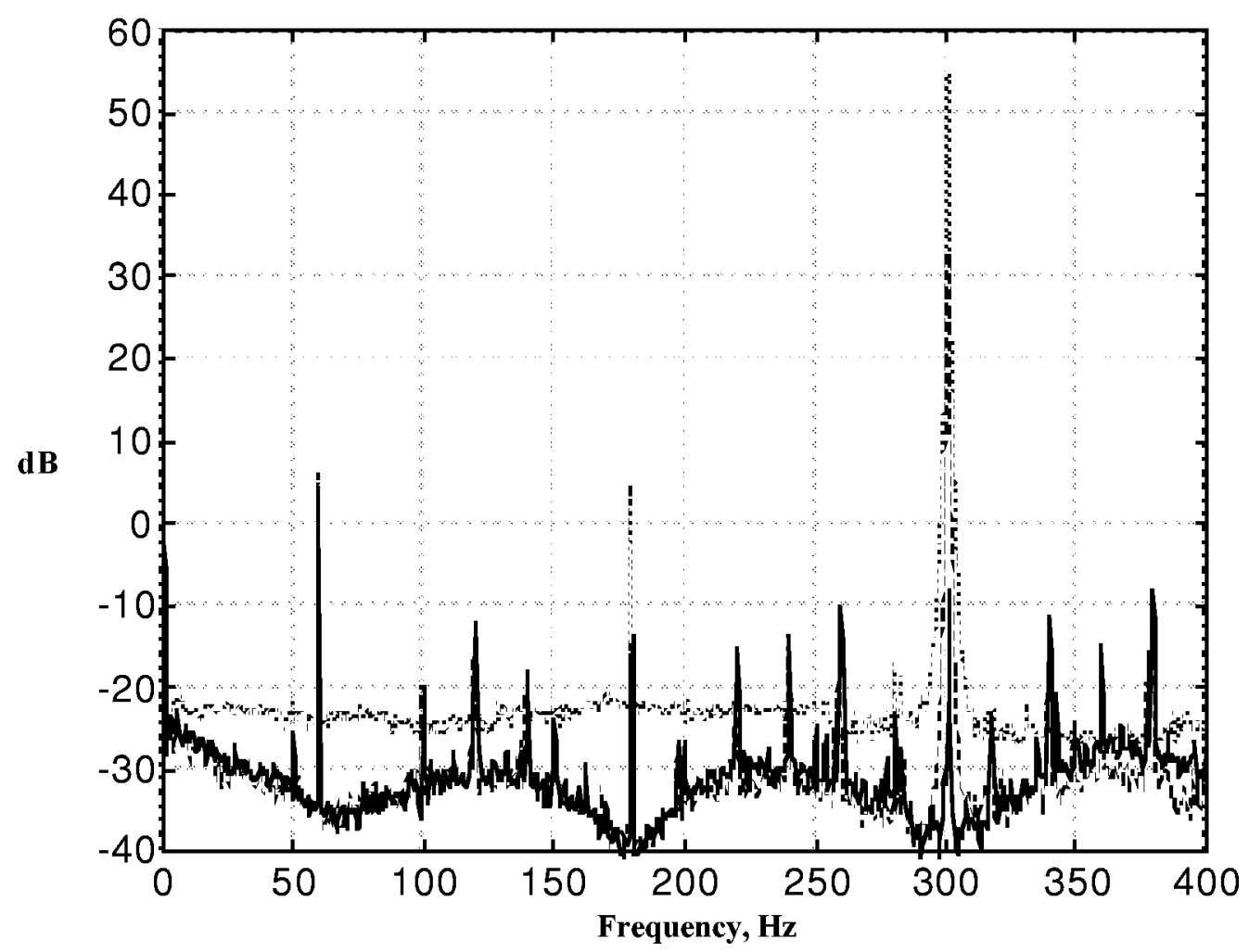

Figure 7: Autospectrum of plant output without control (dotted line), feedback only (dashed line), and feedback with embedded noise model (solid line), 20th order system, $1 \mathrm{KHz}$ sample rate. 


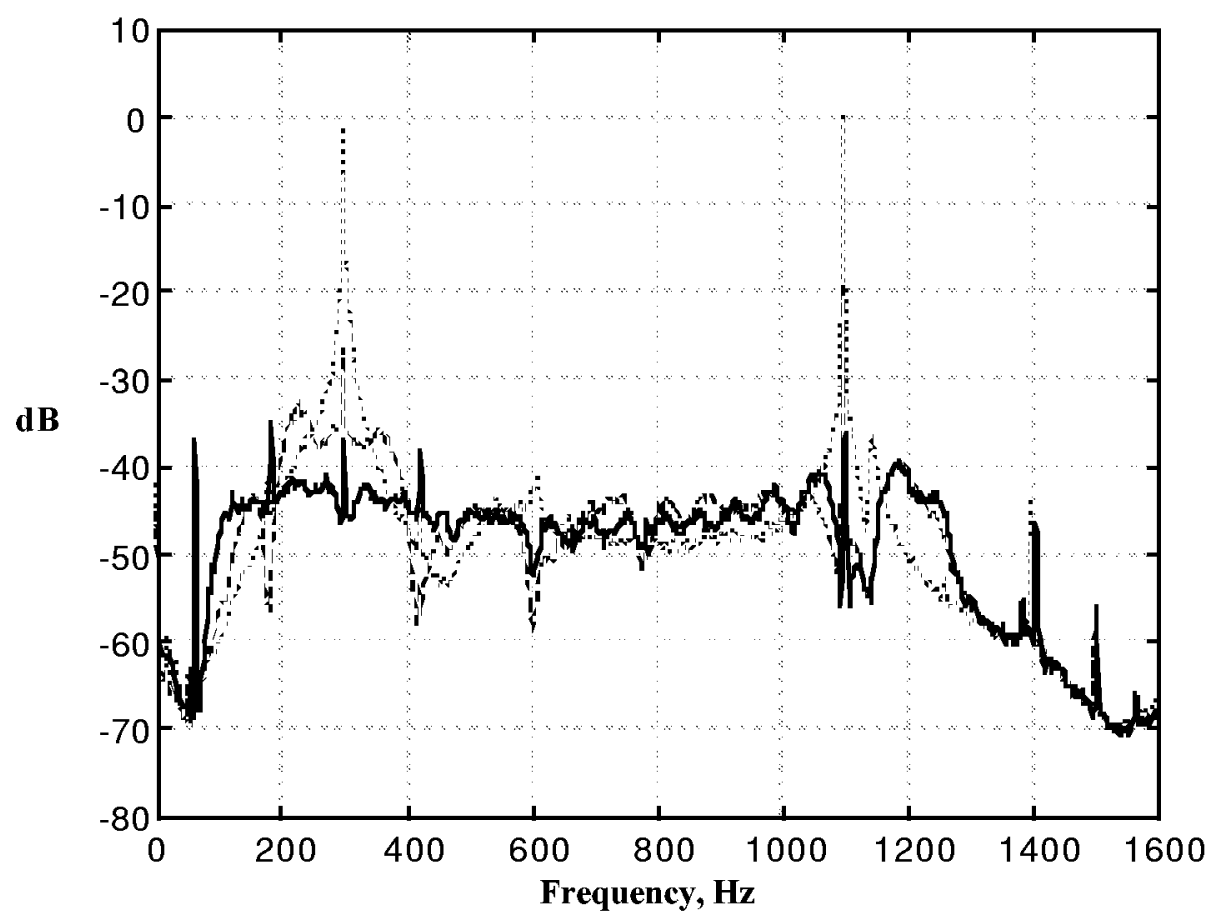

Figure 8: Autospectrum of plant output without control (dotted line), feedback (dashed line), and feedback with embedded noise model (solid line), 50th order system, $2.5 \mathrm{KHz}$ sample rate. 


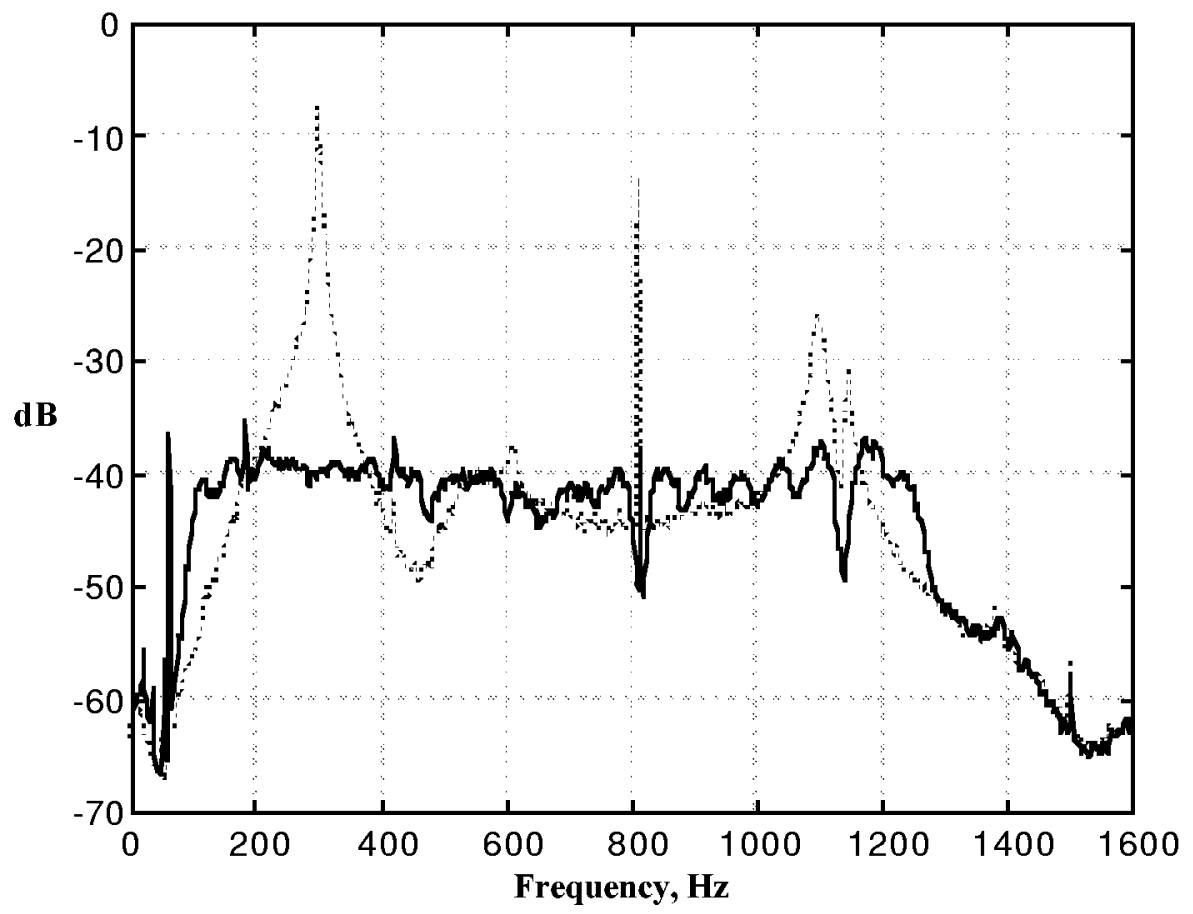

Figure 9: Autospectrum of plant output without control (dotted line) and feedback with embedded noise model (solid line), 50 th order system, $2.5 \mathrm{KHz}$ sample rate. 


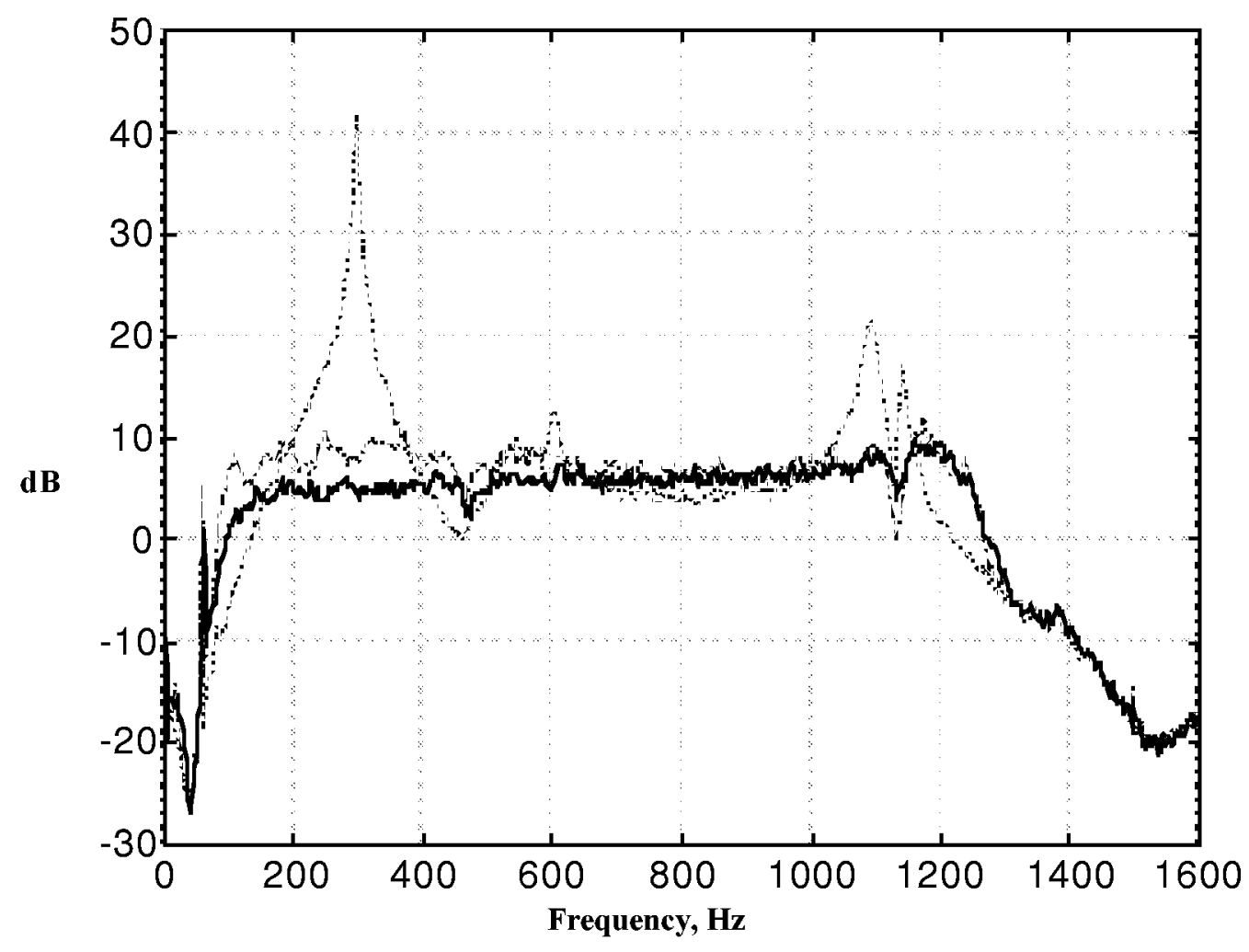

Figure 10: Autospectrum of plant output without control (dotted line), feedback with embedded noise model (dot-dot dash line), and hybrid controller (solid line), 50th order system for all cases, $2.5 \mathrm{KHz}$ sample rate. 


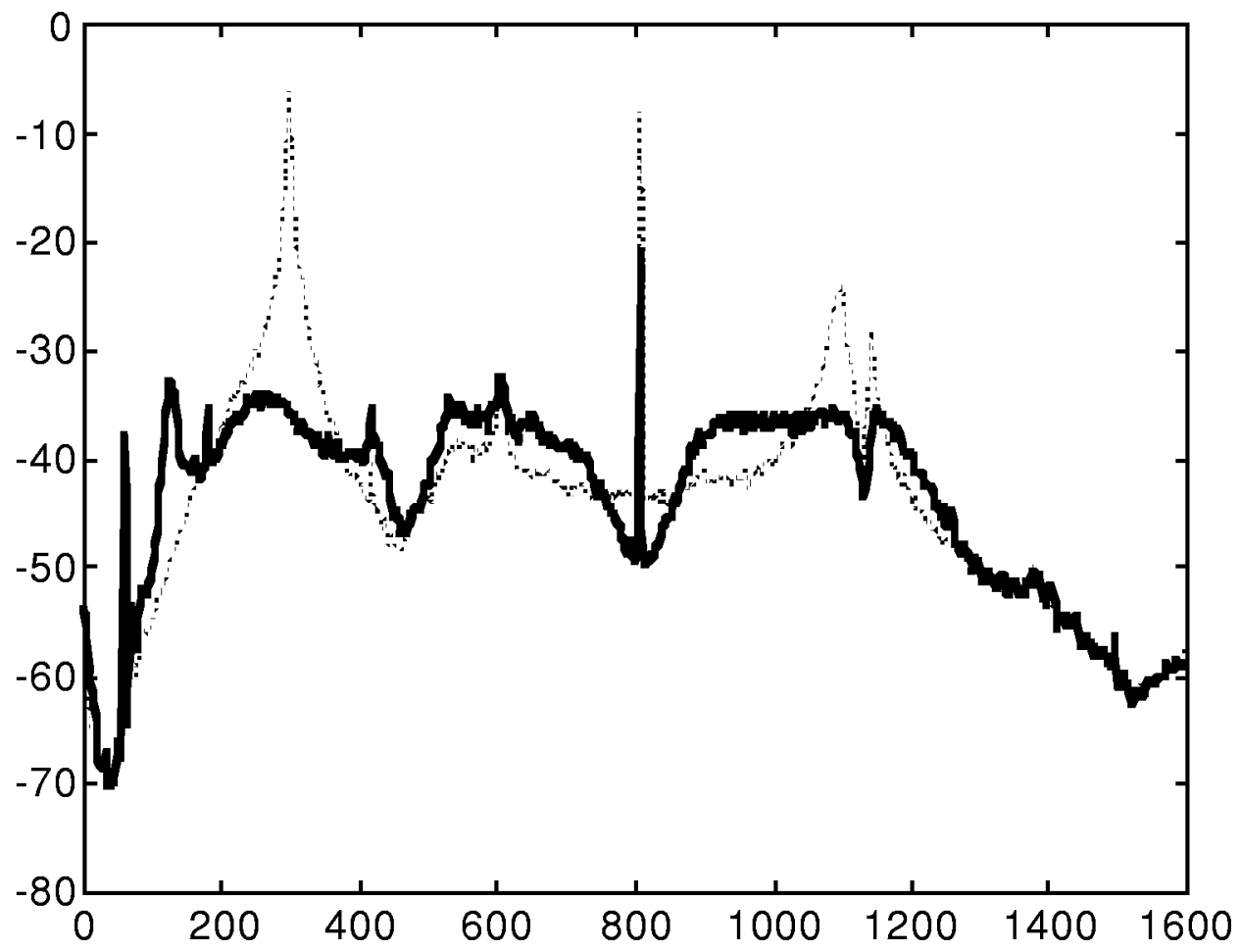

Figure 11: Autospectrum of plant output without control (dotted line), and feedback with embedded noise model (solid line), 14th order system, $2.5 \mathrm{KHz}$ sample rate. 


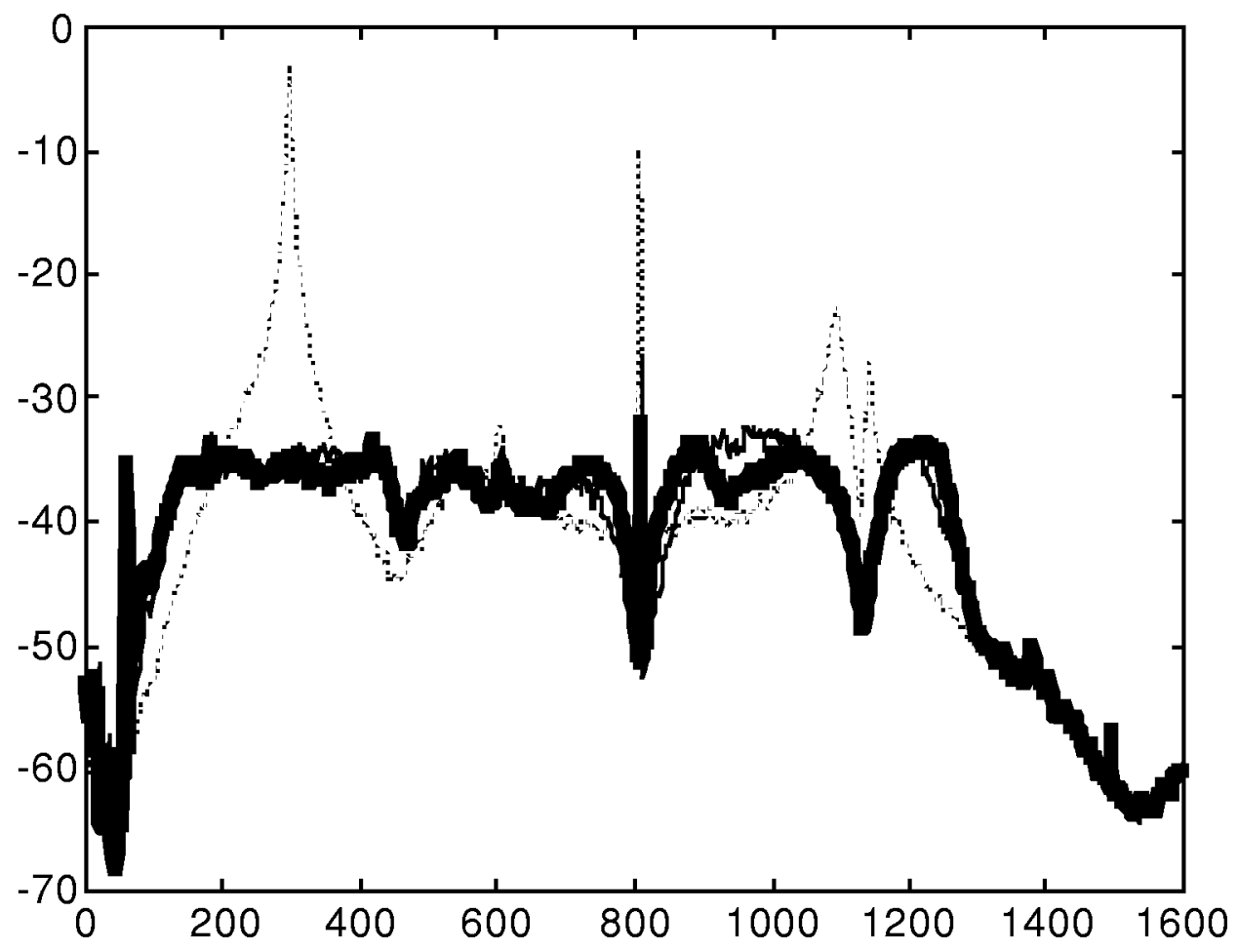

Figure 12: Autospectrum of plant output without control (dotted line), and hybrid control with embedded noise model, 20th order system (thin solid line), 30th order system (thick solid line), $2.5 \mathrm{KHz}$ sample rate. 


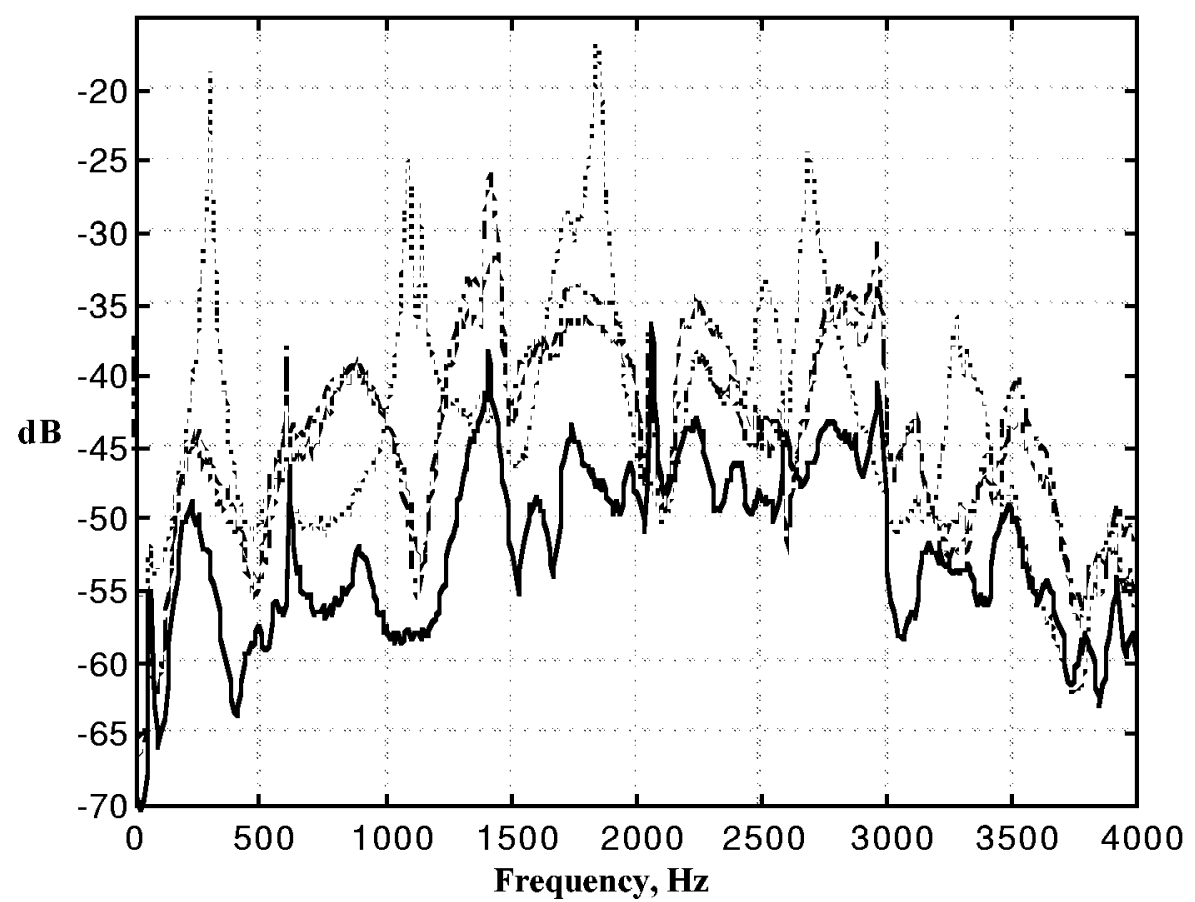

Figure 13: Autospectrum of plant output without control (dotted line), feedback (dot-dot dash line), feedback with embdded noise model (dash-dash dot line), and hybrid control (solid line), 38th order system, sample rate is $10 \mathrm{KHz}$. 


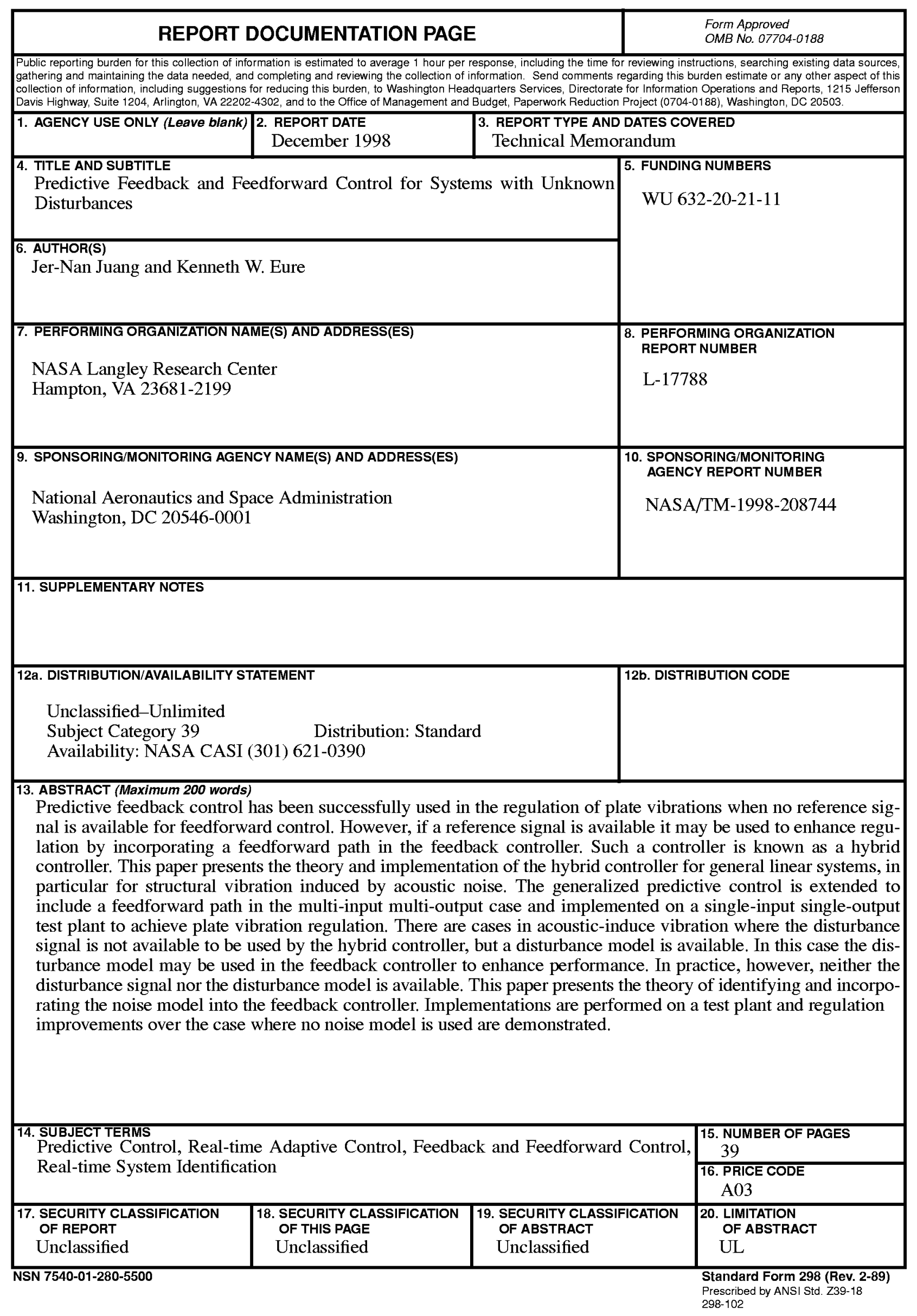

